\title{
La familia Brassicaceae en la provincia de Huaylas, Áncash
}

\author{
The Brassicaceae Family in Huaylas Province, Ancash
}

\author{
Christhian Monsalve y Asunción Cano*
}

Presentado: 26/06/2003

Aceptado: 08/07/2003

\section{Resumen}

Se registró en la provincia de Huaylas un total de 25 especies de Brassicaceae agrupadas en 12 géneros y 5 tribus. Ocho especies se consideran adiciones a la flora previamente reportada para el departamento de Áncash. No se encontró ninguna de las especies reportadas como endémicas para Áncash. Se presentan claves dicotómicas para la determinaciones de los géneros y para las especies en los casos pertinentes. Para cada especie se incluye una breve descripción, datos sobre su ecología, distribución y el material estudiado.

Palabras clave: Brassicaceae, Arabideae, Draba, Perú, endémicas.

\begin{abstract}
A total of 25 species were found in the study area grouped in 12 genera and 5 tribes. Eight species are considered additions to the flora for the department of Ancash. None of the species reported as endemic for Ancash were found. Dichotomous keys are presented for the genera and some species. A brief description and information on the ecology and distribution of each species is included as well as the exicatta.
\end{abstract}

Keywords: Brassicaceae, Arabideae, Draba, Peru, endemic.

\section{Introducción}

La región altoandina es reconocida a nivel mundial como una región de gran interés científico por su diversidad biológica y especialmente por su nivel en endemismos. En el Perú la Cordillera Negra, situada en los Andes occidentales, en el departamento de Áncash, es una zona con vacíos de información botánica (Rodríguez, 1996) y el conocimiento de su flora es incompleto. En el Perú, la familia Brassicaceae es uno de los taxa característicos de ecosistemas altoandinos y sobre la cual se conocen muy pocos estudios taxonómicos o fitogeográficos. Para el departamento de Áncash los estudios precedentes (Smith, 1988; Albán, 1998, Cox, 1998 y Cano et al., 2000) indican que es una familia con un importante número de especies. Antes del presente estudio el registro más extenso para la familia Brassicaceae en Huaylas era el de Albán

* Museo de Historia Natural, Universidad Nacional Mayor de San Marcos, Avda. Arenales 1256, Lima 11, Apartado 14-0434, Lima 14, Perú. Correo-e Christian Monsalve: christianml@hotmail.com
(1998) quien reportó 8 géneros y 11 especies para el distrito de Pamparomás. Según Brako \& Zarucchi (1993), en Áncash se encuentran presentes 17 géneros y 30 especies, de las cuales 8 son endémicas nacionales, lo que la convierte en una de las familias de mayor diversidad y más alto porcentaje de especies endémicas en dicho departamento.

Este trabajo tiene por objetivo presentar los resultados de un estudio taxonómico de la familia Brassicaceae realizado en la provincia de Huaylas. Se espera que sirva de base para un estudio a nivel departamental y nacional.

\section{Área de estudio}

La provincia de Huaylas está ubicada en el departamento de Áncash, al norte del Perú. Mayormente forma parte del extenso valle del Santa, conocido como el "Callejón de Huaylas", y se sitúa entre los $9^{\circ} 20^{\prime}$ y $8^{\circ} 45^{\prime}$ de latitud sur, y $\operatorname{los} 78^{\circ} 10^{\prime}$ y $77^{\circ} 40^{\prime}$ de longitud oeste (Fig. 1).

En la provincia de Huaylas se presentan diversos climas, influenciados grandemente por la altitud. En el fondo del valle el clima es cáli- 


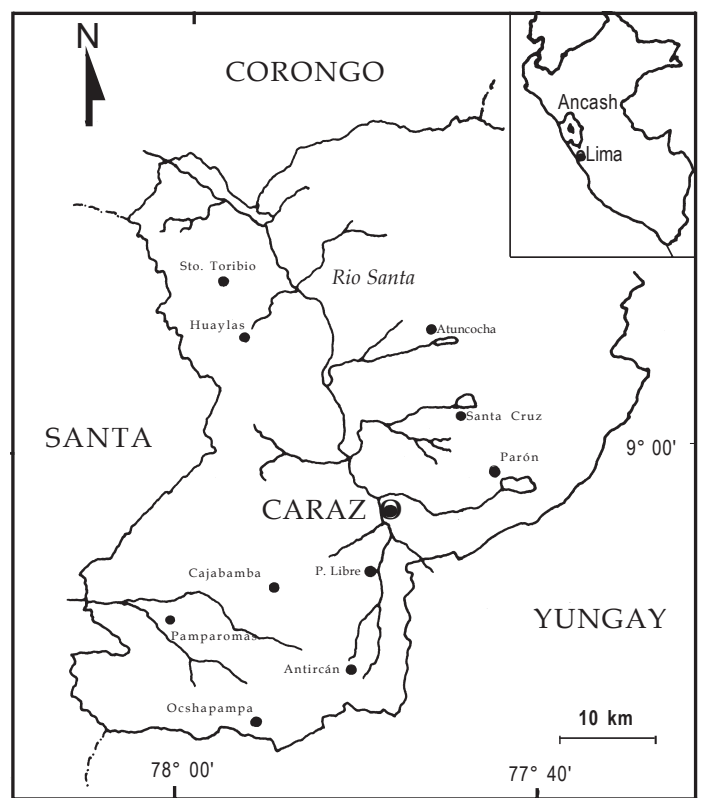

Figura 1. Mapa de la provincia de Huaylas mostrando las principales localidades de colecta.

do y seco la mayor parte del año, con lluvias estacionales entre diciembre y marzo, y excepcionalmente entre noviembre y abril. Conforme se incrementa la altitud sobre los $3200 \mathrm{msnm}$, la temperatura disminuye gradualmente. A grandes altitudes el contraste de temperatura entre el día y la noche o entre el claro y la sombra es notable, se presentan variaciones de hasta más de $20^{\circ} \mathrm{C}$ y las precipitaciones durante los meses de lluvia pueden ser intensas y de larga duración (Peñaherrera, 1969).

\section{Materiales y métodos}

Entre 1999 y 2002 se realizaron colectas en un total de 16 localidades comprendidas en ocho de los 10 distritos de la provincia de Huaylas; se registraron las coordenadas geográficas, altitud y características del ambiente. En el estudio se incluyeron además las colectas realizadas por Smith (1988) en la Cordillera Blanca (Parque Nacional Huascarán), así como las de Carrillo (material de herbario sin fecha de colecta) en Santo Toribio y Albán (1998) en el distrito de Pamparomás, ambas en la Cordillera Negra. Todas estas colecciones se encuentran en el Herbario San Marcos (USM).

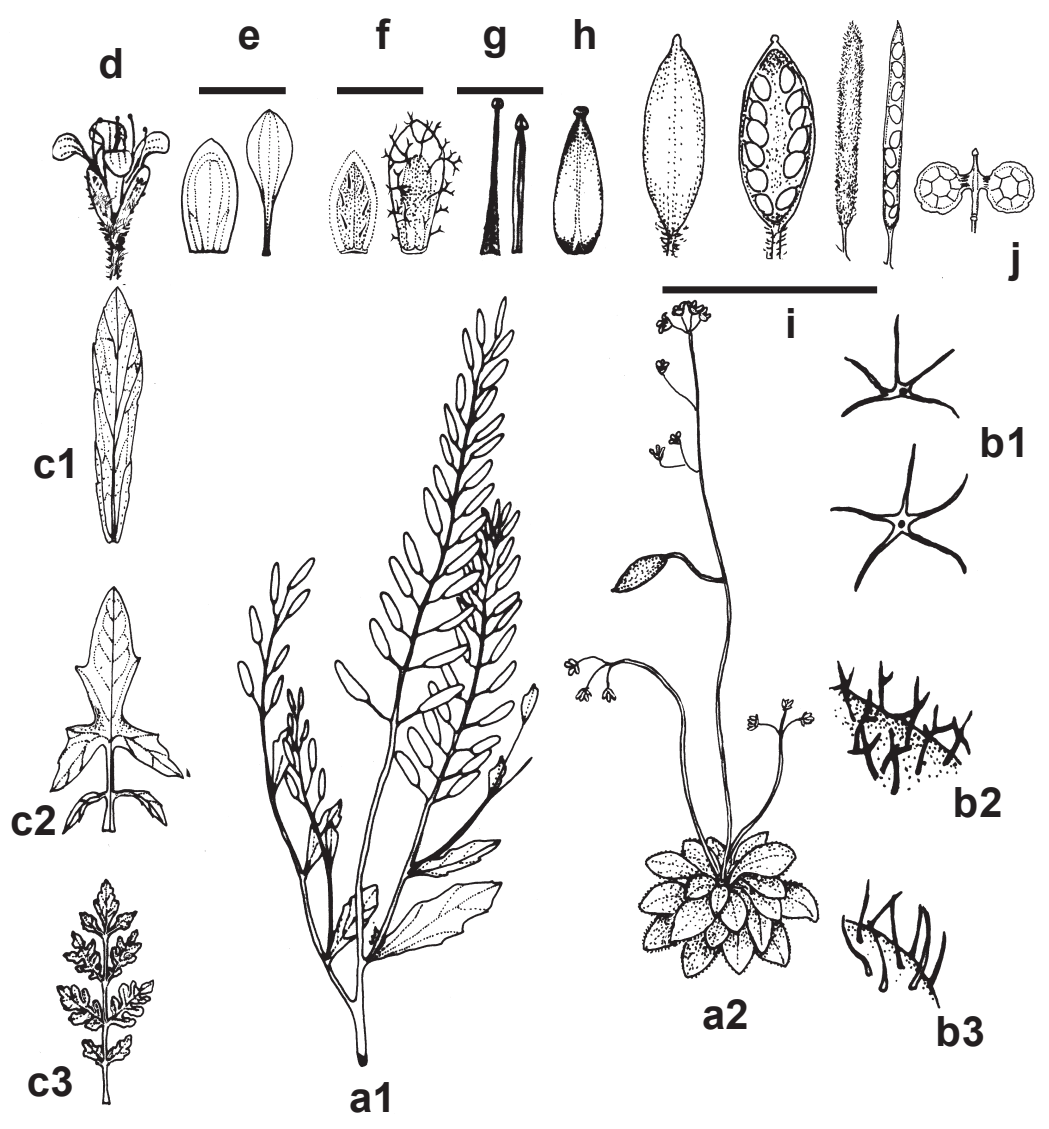

Figura 2. Características generales de la familia Brassicaceae.

a) Hábito: a1. sufrutescente, a2. cespitoso, b) Tricomas: b1. estrellados, b2. bifurcados, b3. simples, c) Hojas: c1. simple, c2. pinnatisecta, c3. bipinnatífida, d) flor tetrámera, e) pétalos, f) sépalos, g) estambres, h) gineceo, i) silícuas, j) silículas 

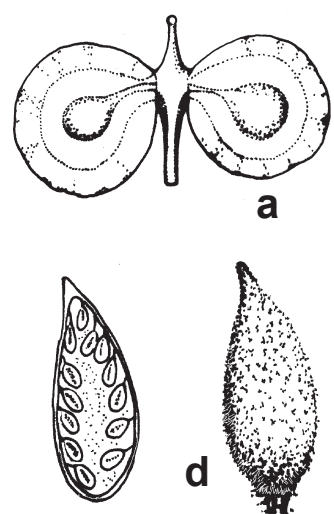

d
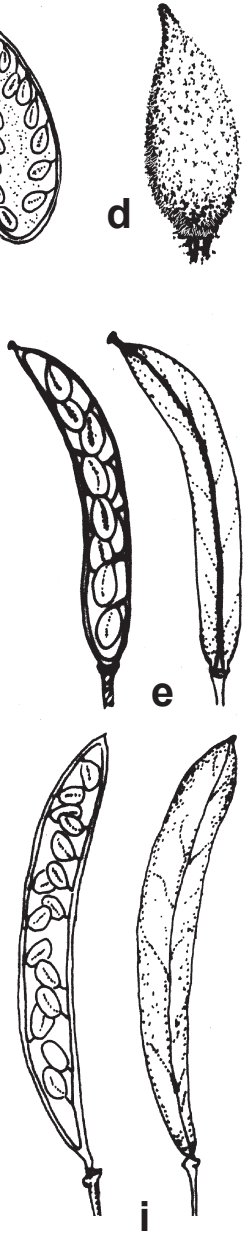
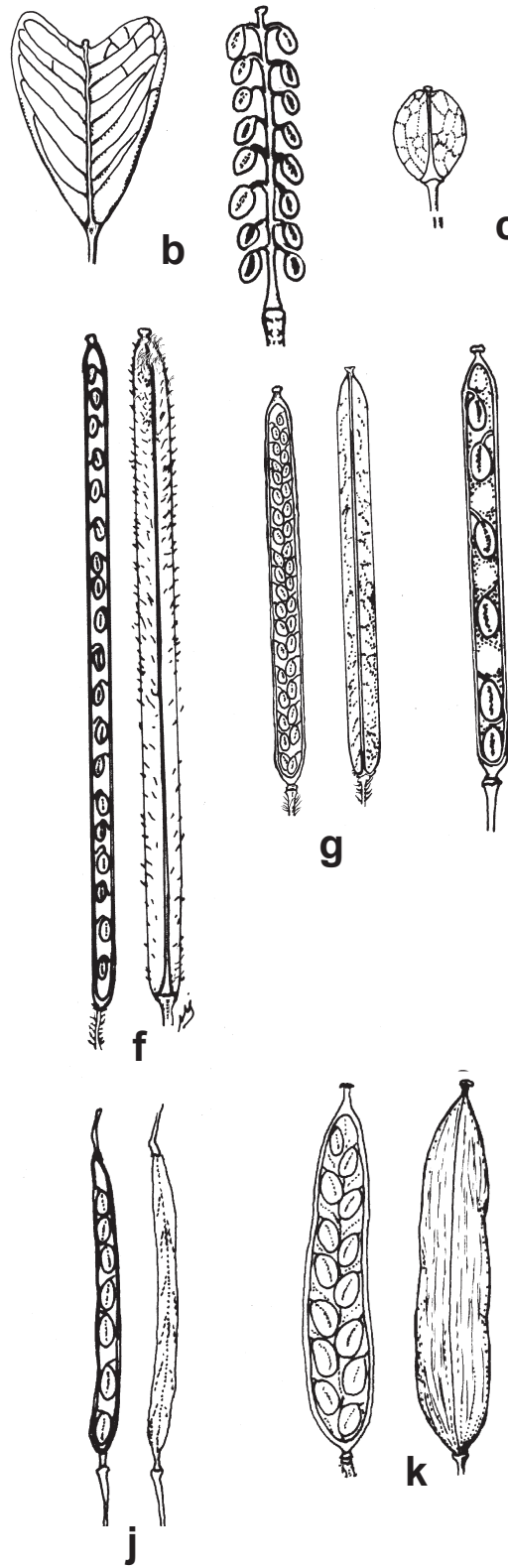
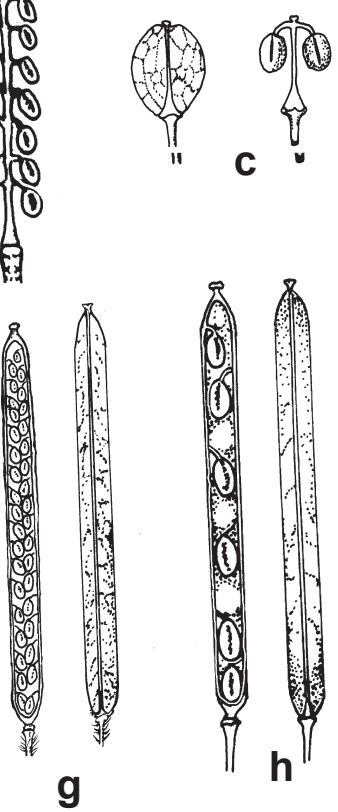

Figura 3. Esquema comparativo de la diversidad morfológica de los frutos entre los géneros de la familia Brassicaceae presentes en Huaylas. (a) Cremolobus, 10x; (b) Capsella, 10x; (c) Lepidium, (10x); (d) Draba, 10x; (e) Brayopsis, 10x; (f) Sisymbrium, 10x; (g) Descurainia, 10x; (h) Cardamine, 10x; (i) Weberbauera, 10x; (j) Brassica, 1x; (k) Rorippa, 10x.
El procesamiento y herborización de las plantas colectadas se realizó siguiendo las técnicas estándares recomendadas (Bridson \& Forman, 1992; Lot y Chiang, 1986 y Cerrate, 1969).

\section{Familia Brassicaceae (= Cruciferae)}

La familia Brassicaceae comprende aproximadamente 350 géneros y 3000 especies, distribuidas en todo el mundo, pero principalmente en las regiones templadas. En el Perú se encuentran registrados 27 géneros y 104 es- pecies, de los cuales 2 géneros y 28 especies son endémicas (Brako y Zarucchi, 1993).

\section{Caracteres taxonómicos de la familia Brassicaceae (Figs. 2, 3)}

Hierbas anuales o perennes, subarbustos o raramente arbustos; glabras o pubescentes, tricomas variados. Tallos típicamente herbáceos o algunas veces sufrutescentes. Hojas de morfología variada, alternas o en ocasiones opuestas; simples; pecioladas o sésiles; sin estípulas. Flores dispuestas en racimos termi- 
Tabla 1 . Diversidad de la familia Brassicaceae en la provincia de Huaylas.

\begin{tabular}{|c|c|c|}
\hline TRIBU & GÉNERO & ESPECIES \\
\hline \multirow[t]{8}{*}{ Arabideae } & Brayopsis & $\begin{array}{l}\text { Brayopsis alpaminae Gilg \& Muschl. } \\
\text { Brayopsis calycina Gilg \& Muschl. }\end{array}$ \\
\hline & Cardamine & Cardamine bonariensis Pers. \\
\hline & & Draba alyssoides Humb. \& Bonpl. \\
\hline & & Draba brackenridgei Gray \\
\hline & Draba & Draba cryptantha Hook. \\
\hline & & Draba cf. hemsleyana Gilg. \\
\hline & & Draba pickeringii Gray \\
\hline & Rorippa & Rorippa nasturtium-aquaticum (L.) Hayek. \\
\hline \multirow[t]{4}{*}{ Brassiceae } & & Brassica juncea (L.) Coss. \\
\hline & Brassica & Brassica nigra (L.) Koch. \\
\hline & & Brassica rapa $\mathrm{L}$ \\
\hline & Raphanus & Raphanus sativus L. \\
\hline Cremolobeae & Cremolobus & Cremolobus chilensis DC. \\
\hline \multirow[t]{5}{*}{ Lepideae } & Capsella & Capsella bursa-pastoris L. \\
\hline & & Lepidium bipinnatifidum Desv. \\
\hline & Lepidium & Lepidium chichicara Desv. \\
\hline & & Lepidium pubescens Desv. \\
\hline & & Lepidium virginicum $\mathrm{L}$. \\
\hline \multirow[t]{6}{*}{ Sisymbrieae } & & Descurainia athrocarpa (Gray) Schulz \\
\hline & Descurainia & Descurainia depressa (Phil.) Reich. \\
\hline & & Descurainia leptoclada Muschl. \\
\hline & & Descurainia myriophylla (Willd.) Fries \\
\hline & Sisymbrium & Sisymbrium peruvianum DC. \\
\hline & Weberbauera & Weberbauera spathulifolia (Gray) Schulz \\
\hline
\end{tabular}

nales, raramente en racimos axilares o solitarias; ebracteadas; hermafroditas, actinomorfas, tetrámeras. Sépalos 4 , dispuestos en dos series; glabros o pubescentes, con bordes hialinos o simples. Pétalos 4, alternisépalos; blancos, amarillos, rojos, lilas o púrpuras; en ocasiones, ausentes. Estambres 6, dispuestos en dos ciclos; tetradínamos; filamentos filiformes, cilíndricos o aplanados hacia la base; anteras con inserción dorsifija, bilobadas, a veces con el conectivo alargado. Nectarios variables en número y disposición, a menudo reunidos formando un anillo alrededor de los estambres. Gineceo con ovario súpero, 2-carpelar, 2locular; estilo simple; estigma capitado o bilobado; óvulos generalmente numerosos, campilótropos. Fruto tipo cápsula, de morfo- logía muy variada; alargado o silicua, o pequeño y comprimido lateralmente o silícula; dehiscente, dejando el replum o septo transversal que separa ambos lóbulos, o indehiscente. Semillas de color variable; superficie lisa o diversamente reticulada; embrión carnoso, rico en aceites; dispuestas en una o dos series en cantidades variables (según Macbride, 1938 y Sagástegui y Leiva, 1993).

\section{Diversidad de la familia Brassicaceae en la provincia de Huaylas}

Se determinó que en la provincia de Huaylas se encuentran presentes 25 especies de la familia Brassicaceae comprendidas en 12 géneros y 5 tribus (Tabla 1). Los géneros 
con mayor diversidad de especies son los géneros Draba con 5 especies y Descurainia y Lepidium, ambos con 4 especies, mientras que los géneros con menor número de especies fueron Capsella, Cardamine, Cremolobus, Raphanus, Rorippa, Sisymbrium y Weberbauera, todos con solamente 1 especie. Por otro lado, la tribu con mayor número de representantes es la tribu Arabideae con 4 géneros y 9 especies y la tribu Cremolobeae es la tribu más pobremente representada con sólo 1 género y 1 especie en la provincia de Huaylas.

De las 25 especies que se encontraron, 8 constituyen nuevos registros para el departamento de Áncash. Los géneros Draba (3) y Lepidium (2) son los que han aportado con un mayor número de nuevos registros. Estos nuevos registros son Descurainia depressa (Phil.) Reich., D. leptoclada Muschl., Draba alyssoides Humb. \& Bonp., D. brackenridgei Gray, D. cf. hemsleyana Gilg, Lepidium pubescens Desv., L. virginicum L. y Sisymbrium peruvianum DC.

Clave para determinar los géneros de la familia Brassicaceae de la provincia de Huaylas

1a. Silícula divididas en dos partes, con ginóforo. ... Cremolobus

1b. Silicuas no divididas en dos partes, raramente con ginóforo.

2a. Plantas glabras o con tricomas alargados simples; silicuas consistentes en porción valvar y estilar.

2b. Plantas pubescentes con tricomas variados; silicuas no consistentes en porción valvar y estilar.

3a. Flores con pétalos generalmente amarillos; silicuas con porción valvar y estilar no muy definidas.

... Brassica

3b. Flores con pétalos blancos o púrpuras; silicuas con porción valvar y estilar marcadamente definidas.

... Raphanus

4a. Plantas herbáceas; silicuas comprimidas lateralmente, septo transversal angosto. 4b. Plantas sufrutescentes o arbustivas; silicuas no comprimidas lateralmente, septo transversal ancho, algunas veces, sufrútices o arbustos.

5a. Hojas ocasionalmente auriculadas; silículas circulares o elípticas. ... Lepidium

5b. Hojas siempre auriculadas; silículas obcordadas.

$$
\text { ... Capsella }
$$

6a. Silicuas anchas, poco más largas que anchas.

... Draba

6b. Silicuas angostas, notablemente más largas que anchas.

7a. Hojas simples o en ocasiones divididas, pero nunca bipinnadas; semillas humedecidas raramente mucilaginosas.

7b. Hojas bipinnadamente partidas; semillas humedecidas frecuentemente mucilaginosas.

... Descurainia

8a. Plantas generalmente perennes, bastante ramificadas; silicuas cortas y delgadas.

8 b. Plantas anuales o bianuales, escasamente ramificadas; silicuas alargadas y anchas.

... Sisymbrium

9a. Plantas anuales, frecuentemente rizomatosas, laxamente ramificadas, herbáceas.

9b. Plantas perennes, nunca rizomatosas, densamente ramificadas, sufrutescentes.

10a. Silicuas desprovistas de nervios; semillas uniseriadas.

... Cardamine

10b. Silicuas nervadas; semillas biseriadas.

... Rorippa

11a. Hojas sésiles; sépalos persistentes.

... Brayopsis

11b. Hojas basales pecioladas; sépalos caducos.

... Weberbauera

\section{Tratamiento de Géneros y especies}

\section{Brassica L.}

Género de origen eurasiático, con 30 especies a nivel mundial, algunas de las cuales son cultivadas o cosmopolitas. En el Perú se encuentran registradas cuatro especies. 


\section{Clave para determinar las especies del género Brassica}

1a. Silicuas de hasta $2 \mathrm{~cm}$ de largo

...B. nigra

1b. Silicuas de más de $3 \mathrm{~cm}$ de largo.

2a. Hojas superiores con base auriculadasemiabrazadora; hojas inferiores lirado-pinnatífidas con lóbulos laterales anchos y grandes; flores en racimos en los que las flores maduras superan en longitud a los botones florales; silicuas de 5-6 cm de largo

....B. rapa

2b. Hojas superiores sésiles pero con base no auriculada o semiabrazadora; hojas inferiores liradopinnatífidas con lóbulos laterales angostos y pequeños; flores en racimos en los que los botones florales superan en longitud a las flores maduras; silicuas de $3-5 \mathrm{~cm}$ de largo

.... B. juncea

\section{Brassica juncea (L.) Cosson}

"Mostaza"

Hierba erguida de tallos angulados y glaucos. Hojas inferiores lirado-pinnatífidas y pecioladas; hojas superiores generalmente sésiles y lanceoladas. Flores amarillas. Silicua glabra con semillas de color marrón oscuro.

Se la encuentra como especie invasora en áreas alteradas por actividad del hombre y zonas de cultivo sobre suelo húmedo y arcilloso.

Material estudiado: Pueblo Libre: Antircán, 4100-4510 msnm, 9 Ago. 2001, C. Monsalve $8 \mathrm{~b}$ (USM).

\section{Brassica nigra (L.) Koch}

"Mostaza, mostaza negra"

Hierba erguida de hasta $1,80 \mathrm{~m}$ de alto, con tallos cilíndricos, híspidos o glabros. Hojas inferiores lirado-pinnatífidas; hojas superiores lanceolado-elípticas. Flores amarillas. Silicua con nervadura central marcada y semillas de color castaño rojizo.

Se la encuentra como especie invasora en áreas alteradas por actividad del hombre y zonas de cultivo.

Material estudiado: Pueblo Libre: Antircán, 4100-4510 msnm, 9 Ago. 2001, C. Monsalve 8c (USM); Cerro Yanaico, 2400-2600 msnm,
12 Abr. 2001, J. Roque 2591 (USM).

\section{Brassica rapa L.}

\section{"Nabo"}

Hierba erguida de tallos cilíndricos generalmente glaucos. Hojas inferiores liradopinnatífidas; hojas superiores oblongolanceoladas. Flores grandes y amarillas. Silicua glabra con semillas redondeadas de color castaño oscuro

Se la encuentra como especie invasora en áreas alteradas por actividad del hombre y zonas de cultivo.

Material estudiado: Huallanca: Tambo, 3900 msnm, D. Smith 11905 (USM). Pueblo Libre: Cerro Yanaico, 2400-2600 msnm, 12 Abr. 2001, C. Monsalve 2 (USM); Cerro Yanaico, 2400-2600 msnm, 12 Abr. 2001, J. Roque 2578 (USM). Pamparomás: Pichiu, 2770 msnm, J. Albán 11299 (USM).

\section{Brayopsis Gilg \& Muschl.}

Género andino con sólo tres especies conocidas las cuales se encuentran registradas para el Perú.

\section{Clave para determinar las especies del género Brayopsis}

1a. Hojas escasamente pubescentes y ovario glabro; pétalos y sépalos subiguales _...B. calycina

1b. Hojas y ovario densamente pubescentes; pétalos notablemente más grandes que los sépalos

...B. alpaminae Bot.

4. Brayopsis alpaminae Gilg \& Muschl

Hierba pulviniforme de tallo corto. Hojas ovado-lanceoladas densamente pubescentes con tricomas simples y largos. Flores solitarias. Silicua elipsoidal y pubescente, con semillas de color castaño oscuro.

Se la encuentra de manera frecuente en pajonales de puna.

Material estudiado: Pamparomás: 
Ocshapampa, 4350-4630 msnm, 11 Oct. 1999, C. Monsalve 4 (USM).

\section{Brayopsis calycina Gilg \& Muschl.}

Hierba cespitosa de tallo corto cubierto por los restos de las bases de las hojas. Hojas ovadolanceolada con tricomas rígidos. Flores solitarias. Silicua con semillas color castaño oscuro.

Se la encuentra de manera frecuente en pajonales de puna.

Material estudiado: Huaylas: Abra de Tres Cruces, 4400-4600 msnm, 18 May. 2000, A. Cano 10470 (USM). Pamparomás: Quebrada Cachicoral, 3900-4400 msnm, 12 Oct. 1999, A. Cano 9794 (USM); Cerro Rico, 4400-4600 msnm, 28 Mar. 2002, C. Monsalve 19 (USM); Cerro Rico, 4400-4600 msnm, 28 Mar. 2002, C. Monsalve 20 (USM); Cerro Rico, 44004600 msnm, 28 Mar. 2002, C. Monsalve 21 (USM). Pueblo Libre: Riurín, 4200-4350 msnm, 18 May. 1999, A. Cano 9139 (USM). Santa Cruz: Lag. Hatuncocha, 4600-4850 msnm, D. Smith 9737 (USM); Pomabamba, 4600-4850 msnm, D. Smith 9952 (USM).

\section{Capsella Medik.}

Comprende cinco especies distribuidas principalmente en regiones templadas a cálidas. En el Perú sólo se registra una especie de distribución cosmopolita.

\section{Capsella bursa-pastoris (L.) Medik.}

"Bolsa del pastor"

Hierba erguida y pubescente con tallos cilíndricos, ramificados desde la base. Hojas basales arrosetadas, enteras o pinnatisectas, pecioladas; hojas superiores lanceoladas, sésiles y sagitadas en la base. Flores blancas o rosadas dispuestas en racimos corimbiformes. Silícula, obdeltoidea, cordada, con semillas de color castaño claro.

Se la encuentra con frecuencia en áreas alteradas por actividad del hombre o eventualmente entre arbustos o pastizales que crecen sobre las laderas rocosas.
Material estudiado: Huallanca: C. Cashacarana, 3900-4000, D. Smith 12098 (USM). Pamparomás: Huachique, 2900 msnm, J. Albán 11465 (USM); Pichiu, 2770 msnm, J. Albán 11589 (USM); Putaca, J. Albán 13693 (USM). Pueblo Libre: Huashta Cruz, 4000-4100 msnm, 29 Mar. 2002, C. Monsalve 26 (USM). Yuramarca: 4020-4700 msnm, D. Smith 9900 (USM).

\section{Cardamine L.}

Comprende alrededor de 130 especies distribuidas en regiones templadas. En el Perú se encuentran presentes cuatro especies.

\section{Cardamine bonariensis Pers.}

\section{"Berro"}

Hierba rizomatosa con tallos decumbentes y raíces adventicias en los nudos. Hojas pinnatipartidas, pecioladas, base auriculada. Flores blancas. Silicua glabra con semillas en números de 20 ó 30 por fruto dispuestas en dos series.

Se la encuentra generalmente como planta emergente enraizada en acequias y pequeños arroyos.

Material estudiado: Pueblo Libre: Antircán, 4100-4510 msnm, 9 Ago. 2001, C. Monsalve 9 (USM). Yuramarca: Quebrada los Cedros, 4020-4050 msnm, D. Smith 9836 (USM).

\section{Cremolobus DC.}

Género típicamente andino, con siete especies conocidas, de las cuales cinco se encuentran en el Perú y una es considerada endémica.

\section{Cremolobus chilensis DC.}

Hierba erguida, hasta de $50 \mathrm{~cm}$ de alto. Hojas generalmente alternas y de morfología variada. Flores blancas con ginóforo. Silícula con valvas generalmente orbiculares, erguidas o pendientes del septo transversal y con una sola semilla por valva. 
Generalmente se la encuentra en matorrales sobre laderas rocosas de pendiente no muy pronunciada.

Material estudiado: Pamparomás: Soukup, 2750 msnm, J. Albán 12853 (USM). Pueblo Libre: Cerro Yanaico, 2350-2800 msnm, 16 May. 1999, A. Cano 8976 (USM).

\section{Descurainia Webb. \& Benth.}

Este género agrupa cerca de 55 especies. Está principalmente distribuido en las regiones templadas y frías del hemisferio norte y sur de África. Para la flora peruana se ha registrado cinco especies.

\section{Clave para determinar las especies del género Descurainia}

1a. Silicuas agrupadas en densos racimos corimbiformes. ... D. athrocarpa

1b. Silicuas agrupadas en racimos alargados.

2a. Silicuas de 4-10 mm de largo .... D. depressa

2b. Silicuas de 10-19 mm de largo.

3a. Hojas con 8 a 10 pares de pinnas.

... D. myriophylla

3b. Hojas con 4 a 6 pares de pinnas .... D. leptoclada

9. Descurainia athrocarpa (Gray) O. E. Schulz

"Alcalde-ccora"

Hierba erguida, pubescente y de tallos cilíndricos. Hojas bipinnatífidas. Flores amarillentas dispuestas en densos racimos corimbiformes. Silicua con semillas de color anaranjado claro dispuestas en una serie.

Se la encuentra preferentemente sobre laderas rocosas en poblaciones no muy numerosas.

Material estudiado: Huaylas: Matacoto, 31003310 msnm, 20 May. 2000, J. Roque 1393 (USM). Pamparomás: Ocshapampa, 4350-4630 msnm, 11 Oct. 1999, A. Cano 9767 (USM). Pueblo Libre: Riurín, 4300-4620 msnm, 19 May.
1999, A. Cano 9239 (USM); Huashta Cruz, 40004200 msnm, 16 May. 2000, A. Cano 10221 (USM). Yuramarca: Quebrada los Cedros, 4020 4050 msnm, D. Smith 9827 (USM).

10. Descurainia depressa (Phil.) Reiche Hierba pubescente con tallos postrados. Hojas bipinnatífidas. Flores blancas o amarillentas, pequeñas. Silicua con semillas de color anaranjado claro dispuestas en una serie.

Se la encuentra preferentemente sobre laderas rocosas en poblaciones no muy numerosas.

Material estudiado: Pamparomás: Cerro Rico, 4400-4600 msnm, 28 Mar. 2002, C. Monsalve 25 (USM).

\section{Descurainia leptoclada Muschl.}

Hierba pubescente de tallos ramificados desde la base. Hojas bipinnatífidas. Flores amarillas. Silicua glabra con semillas dispuestas en una sola serie.

Se la encuentra en gran número sobre laderas rocosas.

Material estudiado: Pamparomás: Cerro Rico, 4400-4600 msnm, 28 Mar. 2002, C. Monsalve 10 (USM); Cerro Rico, 4400-4600 msnm, 28 Mar 2002, C. Monsalve 11 (USM).

12. Descurainia myriophylla (Willd.) Fries

"Hierba de San Juan de Dios"

Hierba pubescente con tallos cilíndricos. Hojas grandes, bipinnatífidas. Flores amarillas. Silicua glabra con semillas de color castaño claro dispuestas en una sola serie.

Se la encuentra preferentemente sobre laderas rocosas en poblaciones no muy numerosas entre arbustos.

Material estudiado: Pamparomás: Carash, 2330 msnm, Albán 11394 (USM); Carash, 2330 msnm, Albán 11634 (USM); Carash, 2330 msnm, Albán 13729 (USM). 


\section{Draba L.}

Es el género más diverso de la familia con alrededor de 300 especies distribuidas en regiones templadas y boreales. En el Perú está representado por 18 especies, de las cuales 9 son endémicas.

\section{Clave para determinar las especies del género Draba.}

1a. Tallos escaposos; plantas cespitosas o pulviniformes.

1b. Tallos no escaposos; plantas generalmente sufrutescentes.

2a. Escapos notablemente largos incluso en flor; hojas sésiles; pétalos más grandes que los sépalos.

2b. Escapos bastante cortos incluso en fruto; hojas pecioladas; pétalos más pequeños o poco más grandes que los sépalos.

... D. cryptantha

3a. Flores con pétalos blancos de $4 \mathrm{~mm}$ de largo; frutos en racimos densos.

... D. pickeringii

3b. Flores con pétalos amarillos menos de $4 \mathrm{~mm}$ de largo; frutos en racimos laxos. … D. brackenridgei

4a. Silicua ovado-elíptica, pubescente.

... D. alyssoides

4b. Silicua ovado-lanceolada, glabra.

... D. cf. hemsleyana

13. Draba alyssoides Humb. \& Bonpl. ex DC.

Hierba erguida e hirsuta de hasta $20 \mathrm{~cm}$ de alto. Hojas oblongo-lanceoladas, sésiles y con uno o dos dientes a cada lado de la lámina. Flores blancas. Silicua ovada con tricomas simples y semillas de color marrón oscuro dispuestas en dos hileras.

Se la encuentra de manera dispersa y escasa en laderas rocosas a la sombra de los arbustos.

Material estudiado: Pueblo Libre: Cerro Yanaico, 2400-2650 msnm, 12 Abr. 2001, A. Cano 11040 (USM).

\section{Draba brackenridgei Gray.}

Hierba pequeña, pubescente y cespitosa con tallo escaposo. Hojas oblongas, dispues- tas en rosetas alrededor del tallo. Flores blancas dispuestas en racimos corimbosos. Silicua ovado-lanceolada con tricomas bifurcados y semillas alargadas de color marrón oscuro dispuestas en dos hileras.

Se la encuentra en laderas rocosas de alta montaña en poblaciones con escasos individuos.

Material estudiado: Pueblo Libre: Riurín, 4200-4350 msnm, 18 May. 1999, A. Cano 9131 (USM); Riurín, 4200-4350 msnm, 18 May. 1999, A. Cano 9137 (USM).

\section{Draba cryptantha Hook.}

Hierba pulviniforme y pubescente con tallos escaposos cortos. Hojas obovadas, dispuestas en densas rosetas. Flores blancas dispuestas en racimos corimbiformes. Silicua pequeña, ovoide, con tricomas simples y semillas de color castaño oscuro.

Se la encuentra en laderas rocosas de alta montaña, en poblaciones con escasos individuos.

Material estudiado: Huaylas: Abra Tres Cruces, 4400-4600 msnm, 18 May. 2000, A. Cano 10456 (USM). Pamparomás: Ocshapampa, 4350-4630 msnm, 11 Oct. 1999, A. Cano 9767 (USM).

\section{Draba cf. hemsleyana Gilg.}

Hierba erguida y pubescente con tallo ramificado. Hojas inferiores oblongas; hojas superiores más pequeñas, linear-oblongas. Flores blancas. silicua ovado-lanceolada, glabra y con semillas dispuestas en dos hileras.

Se la encuentra en laderas rocosas de alta montaña en poblaciones con escasos individuos.

Material estudiado: Caraz: Alrededores Lag, Parón, 4100-4400 msnm, 8 Dic. 1999, M. I. La Torre 2633 (USM).

\section{Draba pickeringii Gray.}

Hierba cespitosa, pubescente y de tallo escaposo. Hojas oblongas. Flores blancas dispuestas en racimos corimbosos. silicua ovoide, pubescente y semillas redondeadas de color marrón oscuro, dispuestas en dos series. 
Se la encuentra en laderas rocosas de alta montaña, en poblaciones con escasos individuos.

Material estudiado: Pueblo Libre: Cerro Rico, 4400-4600 msnm, 28 Mar. 2002, C. Monsalve 12 (USM); Cerro Rico, 4400-4600 msnm, 28 Mar. 2002, C. Monsalve 13 (USM); Cerro Rico, 4400-4600 msnm, 28 Mar. 2002, C. Monsalve 14 (USM).

\section{Lepidium L.}

Este género agrupa cerca de 150 especies. Tiene una distribución cosmopolita, y es especialmente importante en las regiones templadas. En la flora peruana están representadas 14 especies.

\section{Clave para determinar las especies del género Lepidium}

1a. Sépalos caducos; silículas elipsoidales.

1b. Sépalos persistentes; silículas ovadoelipsoidales.

2a. Hojas lirado-pinnatífidas. ... L. virginicum

2a. Hojas enteras. ... L. chichicara

3a. Hojas bipinnatífidas; estilo de $1 \mathrm{~mm}$ de largo. ... L. bipinnatifidum

3b. Hojas pinnatífidas; estilo de 0,3-0,5 mm de largo.

... L. pubescens

18. Lepidium bipinnatifidum Desv. "Mostacilla"

Hierba de tallos postrados dispuestos en roseta. Hojas inferiores bipinnatífidas; hojas superiores binnatipartidas o enteras. Flores blancas en pedúnculos florales recurvados. Silícula ovado-elíptica, con ápice notablemente emarginado y una semilla de color anaranjado claro por valva.

Se la encuentra con frecuencia en áreas alteradas por actividad del hombre, al borde del camino o eventualmente entre arbustos o pastizales que crecen sobre las laderas rocosas.

Material estudiado: Huallanca: C. Cashacarana, 3750-3900 msnm, D. Smith 12004 (USM). Pamparomás: Cerro Rico, 4400-4600 msnm, 28 Mar. 2002, C. Monsalve 17 (USM);
C. Rico, 4400-4600 msnm, 28 Mar. 2002, C. Monsalve 18(USM); Carhuacocha, $4350 \mathrm{msmn}$, J. Albán 11608 (USM); Pamparomás, 2650 msnm, J. Albán 12910 (USM); Putaca, 3100 msnm, J. Albán 12938 (USM). Pueblo Libre: Huashta Cruz, 4000-4200 msnm, 16 May. 2000, A. Cano 10225 (USM); La Hoyada, 3100-3400 msnm, 17 May. 2000, A. Cano 10396 (USM); Cerro Yanaico, 2400-2600 msnm, 12 Abr. 2001, C. Monsalve 6 (USM); Cerro Yanaico, 2400-2600 msnm, 12 Abr. 2001, J. Roque 2643a (USM); Cerro Yanaico, 2400-2600 msnm, 12 Abr. 2001, J. Roque 2643b (USM). Santa Cruz: Quebrada Sta. Cruz, 14 Oct. 1999, A. Cano 9879 (USM).

19. Lepidium chichicara Desv. "Chichicara"

Hierba erguida de tallos ramificados y acanalados. Hojas linear-oblongas y sésiles. Flores blancas. Silícula con una semilla de color anaranjado claro por valva.

Se la encuentra con frecuencia en áreas alteradas por actividad del hombre o eventualmente entre arbustos que crecen sobre las laderas rocosas.

Material estudiado: Huaylas: Huaylas, 4200 msnm, E. Carrillo 1243 (USM). Pueblo Libre: Pueblo Libre, 2300 msnm, 12 Abr. 2001, C. Monsalve 1a (USM); Cerro Yanaico, 24002600 msnm, 12 Abr. 2001, C. Monsalve 7 (USM). Pamparomás: Cajabamba baja, 3200 msnm, J. Albán (s/n); Carhuacocha, 4350 msnm, J. Albán (s/n).

\section{Lepidium pubescens Desv.}

Hierba erguida, escasamente pubescente y con tallos angulados. Hojas inferiores pinnatífidas; hojas superiores pinnatipartidas. Flores blancas. Silícula ovado-elipsoidal con una semilla de color anaranjado claro por valva.

Se la encuentra generalmente en áreas alteradas por actividad del hombre.

Material estudiado: Santo Toribio: Santo Toribio, E. Carrillo 1260 (USM). 


\section{Lepidium virginicum $\mathrm{L}$.}

Hierba erguida, pubescente y de tallos angulados. Hojas lirado-pinnatífidas. Flores blancas. Silícula ovado-elíptica con una semilla alargada de color anaranjado oscuro por valva.

Se le encuentra generalmente en áreas alteradas por actividad del hombre.

Material estudiado: Pueblo Libre: Pueblo Libre, 2300 msnm, 12 Abr. 2001, C. Monsalve $1 \mathrm{~b}$ (USM).

\section{Raphanus L.}

Comprende ocho especies principalmente distribuidas en regiones boreales. En el Perú se encuentran registradas sólo dos especies.

\section{Raphanus sativus L.}

\section{"Rábano"}

Hierba erguida, glabra o pubescente, de tallos poco ramificados. Hojas inferiores liradopinnatipartidas; hojas superiores enteras, sésiles. Flores lilas o grandes. Silicua indehiscente con nervaduras longitudinales y semillas de color castaño.

Se la encuentra como especie invasora o cultivada.

Material estudiado: Pamparomás: Carash, 2350 msnm, J. Albán 11323 (USM). Pueblo Libre: La Hoyada, 2600-3050 msnm, 15 May. 1999, A. Cano 8929 (USM); Antircán, 41004510 msnm, 9 Ago. 2001, C. Monsalve 8a (USM); Cerro Yanaico, 2400-2600 msnm, 12 Abr. 2001, J. Roque 2592 (USM).

\section{Rorippa Scop.}

Comprende alrededor de 70 especies de distribución principalmente cosmopolita. En el Perú se conocen siete especies.

\section{Rorippa nasturtium-aquaticum (L.)} Hayek.

"Berro"
Hierba rizomatosa con tallos ramificados y raíces adventicias en los nudos inferiores. Hojas pinnatisectas con lóbulos de morfología variada. Flores blancas. Silicua globosa, con nerviación reticulada y deprimida entre las semillas. Semilas mucilaginosas en estado húmedo, de color castaño oscuro y superficie reticulada, dispuestas en dos series.

Se la encuentra generalmente como planta emergente enraizada en acequias y pequeños arroyos.

Material estudiado: Pueblo Libre: Pueblo Libre, 2300 msnm, 12 Abr. 2001, Monsalve 3 (USM); Cerro Yanaico, 2400-2600 msnm, 12 Abr 2001, C. Monsalve 5 (USM).

\section{Sisymbrium L.}

Comprende alrededor de 80 especies, de distribución principalmente boreal. En el Perú se encuentran registradas 13 especies, de las cuales seis son endémicas.

24. Sisymbrium peruvianum DC.

Hierba erguida e hirsuta, con tallo muy ramificado. Hojas ovado-elípticas y pecioladas. Flores blancas. Silicua pubescente y recurvada, con semillas de color anaranjado dispuestas en una sola serie.

Se la encuentra preferentemente sobre laderas rocosas, en poblaciones no muy numerosas.

Material estudiado: Pamparomás: Cerro Rico, 4400-4600 msnm, 28 Mar. 2002, C. Monsalve 23 (USM).

\section{Weberbauera Gilg \& Muschl.}

Género andino con siete especies conocidas para el Perú, de las cuales tres son endémicas.

25. Weberbauera spathulifolia (Gray) Schulz.

Hierba postrada de tallos usualmente glabros. Hojas inferiores oblongas y pecioladas; 
hojas superiores pinnatisectas y sésiles. Flores blancas. Silicua glabra, con semillas de color castaño claro dispuestas en dos series.

Se la encuentra sobre laderas rocosas, formando poblaciones dispersas con bajo número de individuos.

Material estudiado: Huaylas: Matacoto, 3100-3310 msnm, 20 May. 2000, J. Roque 1485 (USM); Matacoto, 3100-3310 msnm, 20 May. 2000, J. Roque 1529 (USM). Pamparomás: Cerro Rico, 4400-4600 msnm, 28 Mar. 2002, C. Monsalve 15 (USM); Cerro Rico, 44004600 msnm, 28 Mar. 2002, C. Monsalve 22 (USM); Cerro Rico, 4400-4600 msnm, 28 Mar. 2002, C. Monsalve 24 (USM). Pueblo Libre: Riurín, 4300-4600 msnm, 19 May. 1999, A. Cano 9238 (USM); Huashta Cruz, 4000-4200 msnm, 16 May. 2000, A. Cano 10148 (USM); Huashta Cruz, 4000-4200 msnm, 16 May. 2000, A. Cano 10260 (USM); Hatun Punta, 4250 msnm, 5 Dic 1999, M. I. La Torre 2574 (USM). Yuramarca: C. Alpamayo, 4020-4700 msnm, D. Smith 9924 (USM).

\section{Discusión y conclusión}

Las 25 especies de Brassicaceae que reportamos para la provincia de Huaylas constituyen un incremento significativo al conocimiento de la diversidad de esta familia, considerando los resultados de estudios previos (Smith, 1988; Albán, 1998; Cox, 1998 y Cano et al. 2000).

Es importante resaltar que el número de especies de Brassicaceae registradas en la zona de estudio representa el $83 \%$ del total registrado para la flora peruana para esta familia (Brako \& Zarucchi, 1993).

Se registra 8 especies como nuevas para el departamento de Áncash, aunque entre éstas se encuentren taxa de amplia distribución y algunas conocidas como plantas invasoras o malezas. Cerca del $30 \%$ de las especies registradas suelen encontrarase en los campos de cultivo y zonas aledañas tales como Brassica nigra L., Capsella bursa-pastoris L.,
Lepidium bipinnatifidum Desv. y Rorippa nasturtium-aquaticum (L.) Hayek (Sagástegui \& Leiva, 1993).

Algunos de los nuevos registros están constituidos por especies que fueron encontradas muy alejadas de su rango de distribución conocido (Brako y Zarucchi, 1993) como $D$. depressa y Sisymbrium peruvianum DC, lo cual puede deberse a que se realizaron colectas en lugares anteriormente no visitados o a que simplemente el rango de distribución de tales especies se ha ampliado, gracias al transporte de las semillas posiblemente a través del pastoreo. Sin embargo, los nuevos registros más interesantes están constituidos por especies del género Draba, que aporta con tres especies (D. alyssoides, D. brackenridgei y $D$. cf. hemsleyana), las cuales fueron colectadas en sólo una localidad y en un número muy reducido de individuos.

No se encontró en Huaylas ninguna de las especies consideradas endémicas para el departamento de Áncash por Brako \& Zarucchi (1993). La mayoría de estas especies, como Eudema incurva Al-Shehbaz y Dactylocardamum imbricatifolium Al-Shehbaz (el último, género monoespecífico), sólo han sido colectadas una sola vez en la respectiva localidad tipo y ningún duplicado se encuentra depositado en herbarios nacionales. Tales ausencias pueden deberse a que algún factor ambiental esté limitando su distribución a sólo unas pocas localidades, o a que se trate de especies cuyos individuos se desarrollan de manera muy aislada, sin formar extensas poblaciones, lo que podría haber dificultado su reconocimiento en el campo, tal y como se ha observado con las especies del género Draba incluidas en este estudio, con excepción de $D$. cryptantha que sí pudo ser colectada en varias localidades. También podría deberse a que son especies en vías de extinción o extintas.

Podemos concluir en que la provincia de Huaylas (Áncash) tiene una alta diversidad de Brassicaceae con 25 especies, comprendidas en 12 géneros y 5 tribus y que representan el 
$83 \%$ del total de especies de esta familia para la flora peruana.

\section{Agradecimientos}

Los autores agradecen por el apoyo económico al Fondo de Desarrollo Universitario (FEDU) de la Universidad Nacional Mayor de San Marcos y a National Geographic Society (Grant N. ${ }^{\circ} 6407-99$ al proyecto "Plant diversity and endemism in Peru's central highlands"). Nuestra inmensa gratitud a los colegas Joaquina Albán, Magda Chanco, María I. La Torre, Blanca León, Mery Suni, José Roque y Kenneth R. Young por la revisión y sugerencias al manuscrito. También a Marissa Ocrospoma por las ilustraciones.

\section{Literatura citada}

Albán, J. 1998. Etnobotánica y conservación en la comunidad andina de Pamparomás, Huaylas, Áncash, Perú. Tesis para optar el grado académico de Magíster. UNMSM.

Brako, L.; J. Zarucchi. 1993. Catálogo de las Angiospermas y Gimnospermas del Perú.

Bridson, D \& L. Forman. 1992. Herbarium Handbook. Royal Botanic Gardens, Kew.

Cano, A.; K. R. Young y B. León. 1996. Áreas importantes para la conservación de las fanerógamas en el Perú. pp. 39-46 en Rodríguez, L. Diversidad biológica del Perú: Zonas Prioritarias para su Conservación. Proyecto FANPE, GTZINRENA, Lima.
Cano, A.; M. Suni, K. R. Young, B. León, M. I. La Torre, J. Roque y A. Ramírez. 2000. Diversidad florística y endemismos en las Cordilleras Blanca y Negra (Áncash). Sector Bajo. Informe Técnico Final presentado al CSI y al ICBAR.

Cerrate, E. 1969. Manera de preparar plantas para un herbario. Museo de Historia Natural. Serie de Divulgación N. ${ }^{\circ} 1$.

Cox, E. 1998. Estudio Etnobotánico de los Pueblos de Huaylas y Santo Toribio (Áncash). Tesis para optar el grado de Magíster. UNMSM.

Lot, E. y F. Chiang (Compiladores). 1986. Manual de Herbario. Consejo Nacional de la Flora de México. México. 42 pp.

Macbride, J. F. 1938. Flora of Peru, part 1. Fieldiana: Bot. 13 (1): 1-170.

Peñaherrera, C. 1969. Geografía General del Perú. Tomo I. Aspectos Físicos.

Rodríguez, L. (ED.). 1996. Diversidad biológica del Perú: Zonas Prioritarias para su Conservación. Proyecto FANPE, GTZ-INRENA, Lima.

Sagástegui, A. y S. Leiva. 1993. Flora invasora de los cultivos del Perú. Editorial Libertad, EIRL. Trujillo.

Smith, D. 1988. Flora and Vegetation of the Huascaran National Park, Ancash, Peru; with preliminary studies for a manual of the flora. Thesis to obtain the degree of Doctor of Philosophy. Iowa State University, Ames, Iowa. 